\title{
Retraction Note to: Downregulation of miR-148b as biomarker for early detection of hepatocellular carcinoma and may serve as a prognostic marker
}

\author{
Katayoun Ziari ${ }^{1}$ - Mojtaba Zarea ${ }^{2}$ - Masoumeh Gity ${ }^{3}$. Amir Farshid Fayyaz ${ }^{4}$. \\ Emad Yahaghi ${ }^{5}$ - Ebrahim Khodaverdi Darian ${ }^{6} \cdot$ Amir Masoud Hashemian ${ }^{7}$
}

Published online: 5 November 2016

(C) International Society of Oncology and BioMarkers (ISOBM) 2016

\section{Retraction Note to: Tumor Biol (2016) 37:5765-5768 \\ DOI 10.1007/s13277-015-3777-4}

This article has been retracted at the request of the Editor-in-Chief, the International Society of Oncology and BioMarkers (ISOBM) and the Publisher per the Committee on Publication Ethics guidelines. The article shows evidence of irregularities in authorship during the submission process, there is strong reason

The online version of the original article can be found at http://dx.doi. org/10.1007/s13277-015-3777-4.

Amir Masoud Hashemian

hashemianam@mums.ac.ir

Department of Pathology, Be'sat Hospital, AJA University of Medical Sciences, Tehran, Iran

2 Center for Chemical Biology, Indian Institute of Chemical Technology (IICT), Tarnaka, Hyderabad, India

3 Department of Radiology, Medical Imaging Center, Tehran University of Medical Sciences, Tehran, Iran

4 Department of Legal Medicine, AJA University of Medical Sciences, Tehran, Iran

5 Baqiyatallah University of Medical Sciences, Tehran, Iran

6 Young Researchers and Elite Club, Karaj Branch, Islamic Azad University, Karaj, Iran

7 Department of Emergency Medicine, Imam Reza Hospital, Faculty of Medicine, Mashhad University of Medical Sciences, Mashhad, Iran to believe that the peer review process was compromised and there are similarities with the following articles which were all submitted within a close timeframe:

Naeemeh Ghasemkhani, Sahar Shadvar, Yasamin Masoudi, Amir Jouya Talaei, Emad Yahaghi, Peyman Karimi Goudarzi, Ebrahim Shakiba, Down-regulated MicroRNA $148 \mathrm{~b}$ expression as predictive biomarker and its prognostic significance associated with clinicopathological features in non-small-cell lung cancer patients. Diagnostic Pathology 2015, 10:164 DOI: 10.1186 /s13000-015-0393-y

Date received: 18 June 2015

Yasan Sadeghian, Zahra Kamyabi-Moghaddam, Seyed Mohamad Hossein Tabatabaei Nodushan, Samaneh Khoshbakht, Behnam Pedram, Emad Yahaghi, Aram Mokarizadeh, Mahdi Mohebbi, Profiles of tissue microRNAs; miR-148b and miR-25 serve as potential prognostic biomarkers for hepatocellular carcinoma. Tumor Biol. First Online: 25 July 2015 DOI: 10.1007 /s13277-015-3799-y

Date received: 1 July 2015

The retracted article was received: 16 June 2015

As such the validity of the content of this article cannot be verified. 